\title{
SARKOVSKII'S THEOREM FOR HEREDITARILY DECOMPOSABLE CHAINABLE CONTINUA
}

\author{
PIOTR MINC AND W. R. R. TRANSUE
}

ABSTRACT. Sarkovskii's theorem, which fails to hold for chainable continua, is shown to hold for hereditarily decomposable chainable continua.

\section{INTRODUCTION}

In 1964 A. N. Sarkovskii [8] introduced the following ordering of the positive integers: $1 \triangleleft 2 \triangleleft 4 \triangleleft \cdots \triangleleft 2^{k} \triangleleft 2^{k+1} \triangleleft \cdots \triangleleft 2^{k+1} 7 \triangleleft 2^{k+1} 5 \triangleleft 2^{k+1} 3 \triangleleft \cdots \triangleleft 2^{k} 7 \triangleleft 2^{k} 5 \triangleleft$ $2^{k} 3 \triangleleft \cdots \triangleleft 14 \triangleleft 10 \triangleleft 6 \triangleleft \cdots \triangleleft 9 \triangleleft 7 \triangleleft 5 \triangleleft 3$. Sarkovskii proved that if $m \triangleleft n, f$ is a continuous map of an interval into the real line and $f$ has a periodic point of period $n$, then $f$ has a point of period $m$. The object of this paper is to show that the Sarkovskii theorem holds for hereditarily decomposable chainable continua. We shall prove the following theorem.

Theorem 1.1. Let $Y$ be a hereditarily decomposable chainable continuum and let $X$ be a subcontinuum of $Y$. If $m \triangleleft n, f$ is a continuous map of $X$ into $Y$ and $f$ has a periodic point of period $n$, then $f$ has a point of period $m$.

By a continuum we mean a compact connected metric space. A continuum is decomposable if it can be written as the union of two proper subcontinua. It is hereditarily decomposable if each of its nondegenerate subcontinua is decomposable. A continuum $Y$ is chainable if for each positive number $\varepsilon$, there is an $\varepsilon$-map $h$ of $Y$ onto $[0,1]$, that is, a map whose point inverses have diameter less than $\varepsilon$. If $I \subset X \subset Y$ and $f: X \rightarrow Y$ is a map, we can define $f^{n}(I)$ inductively as $f\left(X \cap f^{n-1}(I)\right)$. Observe that $f^{n}(I)$ may be empty and that it will be a continuum if $I$ is a continuum and $Y$ is chainable. If $x$ is a point, using the notation $f^{n}(x)$ will imply that $x, f(x), f^{2}(x), \ldots, f^{n-1}(x)$ are points of $X$ and $f^{n}(x)$ is a properly defined point of $Y$. The period of a point $x$ under a map $f$ is the least positive integer $n$ so that $f^{n}(x)=x$.

The question was raised by Marcy Barge, who has informed us (in private communication) of partial results in addition to examples showing the necessity of the assumption of hereditary decomposability. We describe briefly here an

Received by the editors March 10, 1988.

1980 Mathematics Subject Classification (1985 Revision). Primary 54F20, 58F20.

Key words and phrases. Sarkovskii's theorem, periodic point, chainable, hereditarily decomposable. 
example of a map of a chainable (indecomposable) continuum into itself having a point of period 3 but none of period 2, and then proceed with the proof of the theorem. A better example was given earlier by Wayne Lewis [6], who showed that for every $n$ there is a chainable continuum with a periodic homeomorphism of period $n$. The general outline of the proof of Theorem 1.1 is patterned on that of Block, Guckenheimer, Misiurewicz and Young [1] as described in Devaney's book [2].

In the proof we shall use diagrams with sets and arrows (sometimes labeled). The meaning of such a diagram is that each set at the tail of an arrow is mapped by the function labeling the arrow (or if there is no label, $f$ ) onto a set containing the set at the head of the arrow. In the proof of the Sarkovskii theorem on an interval the sets in question are intervals and typically overlap at most in a point whose periodicity is known. The fixed point theorem for maps of an interval onto an interval containing it can then be used to infer the existence of points of other periods. Thus, for example, if in the diagram $f \subset A \underset{f^{2}}{\stackrel{f}{\leftrightarrows}} B, A$ and $B$ were intervals having only a point of period 5 in common, one could easily conclude the existence of points of periods $3,4,6,7, \ldots$ In our proof the spaces are, instead of intervals, chainable continua. In 1951 O. H. Hamilton [3] showed that chainable continua have the fixed point property. The proof readily shows that a map from a subcontinuum of a chainable continuum $Y$ onto $Y$ has a fixed point. For example this theorem allows to infer the existence of points of all periods from the diagram $C A \leftrightarrows B$ if $A$ and $B$ are disjoint chainable continua. Our difficulties arise because of the considerable overlaps which confront us. In [4] the same diagram was studied under an additional assumption about the behavior of the function on the intersection of $A$ and $B$. In the case of Theorem 1.1 such assumption is not available. Our way out is frequently to get more complicated diagrams in which the spaces do not overlap.

Example 1.2. An example of a map of a chainable (indecomposable) continuum into itself having a point of period 3 but none of period 2 .

Let

$$
f(x)= \begin{cases}2 x & \text { for } 0 \leq x \leq \frac{1}{2} \\ 2-2 x & \text { for } \frac{1}{2} \leq x \leq 1\end{cases}
$$

Similarly let

$$
g(x)= \begin{cases}15 x & \text { for } 0 \leq x \leq \frac{1}{15} \\ 2-15 x & \text { for } \frac{1}{15} \leq x \leq \frac{2}{15} \\ 15 x-2 & \text { for } \frac{2}{15} \leq x \leq \frac{3}{15} \\ 4-15 x & \text { for } \frac{3}{15} \leq x \leq \frac{4}{15} \\ \ldots \ldots \ldots \ldots \ldots \ldots \ldots \ldots \ldots \ldots \ldots \ldots \ldots \ldots \ldots \ldots \ldots & \ldots \ldots \ldots \ldots \\ \ldots \ldots \ldots \ldots & \text { for } \frac{14}{15} \leq x \leq 1 \\ 15 x-14\end{cases}
$$


Let $M$ be the inverse limit $[0,1] \stackrel{g}{\longleftarrow}[0,1] \stackrel{g}{\leftarrow} \ldots . f$ and $g$ commute. Let $\tilde{f}$ be the map induced by $f$ on $M$. The point $p=\left(\frac{2}{7}, \frac{2}{7}, \frac{2}{7}, \ldots\right)$ is in $M$. Since $f^{3}\left(\frac{2}{7}\right)=f^{2}\left(\frac{4}{7}\right)=f\left(\frac{6}{7}\right)=\frac{2}{7}, p$ has period 3 under $\tilde{f}$, any point of period 2 under $\tilde{f}$ would have to have each of its coordinates fixed under $f^{2}$ and one of them not fixed under $f$. The fixed points of $f^{2}$ are $0, \frac{2}{5}, \frac{2}{3}$ and $\frac{4}{5}$. But since $g\left(\frac{4}{5}\right)=g\left(\frac{2}{3}\right)=g\left(\frac{2}{5}\right)=g(0)=0$, the only point of $M$ fixed under $\tilde{f}^{2}$ is $(0,0,0, \ldots)$, which is the fixed point of $\tilde{f}$.

\section{HEREDITARILY DECOMPOSABLE IRREDUCIBLE CONTINUA}

We shall assume some familiarity with Chapter V, $\S 48$ of [5]. In particular given a hereditarily decomposable continuum $M$ irreducible between two points $a$ and $b$, there is a continuous function $g$ from $M$ onto $[0,1]$ with $g(a)=0, g(b)=1$ and $g^{-1}(t)$ a maximal nowhere dense subcontinuum for each $t$ in $[0,1]$. We shall call $g$ a Kuratowski function. Subcontinua $g^{-1}(t)$ are called layers of $M ; g^{-1}(0)$ and $g^{-1}(1)$ are called end layers of $M$; the other layers are called interior layers. If $x$ and $y$ are points of $M$ we denote by $[x, y]$ a continuum irreducible between $x$ and $y$ (in the sequel, when $M$ is chainable, $[x, y]$ will be unique). Denote $[x, y]$ minus its end layers by $(x, y)$. Denote also $[x, y]$ minus its layer containing $y$ (or $x$ ) by $[x, y$ ) (or $(x, y]$, respectively).

Lemma 2.1. Let $f: Z \rightarrow C$ be a continuous mapping of a hereditarily decomposable irreducible continuum $Z$ onto a hereditarily decomposable irreducible continuum $C$. Let $A$ and $B$ be the end layers of $Z$ and let $L$ be an end layer in $C$. If $L$ is disjoint with the image of $Z-(A \cup B)$ and there is a point $a \in A$ such that $f(a) \in L$, then $L \subset f(A)$.

Proof of Lemma 2.1. Since $L$ is an end layer of $C$, it is contained in every subcontinuum of $C$ intersecting both $L$ and $C-L$. It follows that $f([a, z])$ contains $L$ for every $z \in[a, b]-A$. Since $A$ is the intersection of all $[a, z]$ with $z \in[a, b]-A$, we have that $L \subset f(A)$.

Lemma 2.2. Let $f: Z \rightarrow C$ be a continuous mapping of a hereditarily decomposable irreducible continuum $Z$ onto a hereditarily decomposable irreducible continuum $C$. Let $A$ and $B$ be the end layers of $Z$. If $c$ is a point belonging to an interior layer of $C$ then there is a point $z \in Z-(A \cup B)$ such that $f(z)=c$.

Proof of Lemma 2.2. Let $L$ be the layer of $c$ in $C$. Since $L$ is an interior layer of $C, C-L$ has two components, let us say, $U$ and $V$. Since $Z-(A \cup B)$ is dense in $Z$, there are points $u, v \in Z-(A \cup B)$ such that $f(u) \in U$ and $f(v) \in V$. The lemma follows from the inclusion $L \subset[f(u), f(v)]$.

Lemma 2.3. Let $f: Z \rightarrow C$ be a continuous mapping of a hereditarily decomposable irreducible continuum $Z$ onto a hereditarily decomposable irreducible continuum $C$. Suppose that $Z=[a, b]$ and $L$ is an end layer in $C$. If there 
is a point $p \in[a, b)$ such that $f(p) \in L$, then there is a point $q \in(a, b)$ such that $L \subset f([p, q])$.

Proof of Lemma 2.3. Since $(a, b)$ is dense in $Z$ and $L$ is nowhere dense in $C$, there is a point $q \in(a, b)$ such that $f(q) \notin L$. Since $L$ is an end layer of $C$, it is contained in every subcontinuum of $C$ intersecting both $L$ and $C-L$. In particular it is contained in $[f(p), f(q)] \subset f([p, q])$.

Lemma 2.4. Let $f: Z \rightarrow C$ be a continuous mapping of a hereditarily decomposable irreducible continuum $Z$ onto a hereditarily decomposable irreducible continuum $C$. Let $A$ and $B$ be the end layers of $Z$. Let $a, b$ and $c$ be three points from $A, B$ and $C$, respectively. Then the following two statements are true.

(i) If $c \in[f(a), f(b))$, then there is a point $t \in[a, b)$ such that $f(t)=c$.

(ii) If $c \in(f(a), f(b))$ then either

$$
\begin{aligned}
& \text { there is a point } t \in(a, b) \text { such that } f(t)=c \text { or } \\
& {[f(a), f(b)] \subset f(A) \cap f(B) .}
\end{aligned}
$$

Proof of Lemma 2.4. Let $L$ be the layer of $c$ in $f([a, b])$. We can assume that $L$ is an end layer of $f([a, b])$, because otherwise the lemma follows from Lemma 2.2. Applying Lemma 2.3 we can also assume that no point of $(a, b)$ is mapped into $L$.

Since $c \in[f(a), f(b)]$, we have that at least one of the points $f(a)$ and $f(b)$ belongs to $L$. If $f(a) \notin L$, then $f(b) \in L$ and $L$ coincides with the layer of $f(b)$ in $[f(a), f(b)]$. So if $c \in[f(a), f(b))$, then $f(a) \in L$ and it follows from Lemma 2.1 that $c \in f(A)$. If $f(b) \notin L$, then $f(a) \in L$ and $L$ coincides with the layer of $f(a)$ in $[f(a), f(b)]$. So if $c \in(f(a), f(b))$, then both $f(a)$ and $f(b)$ belong to $L$ and the lemma follows from Lemma 2.1.

Lemma 2.5. Let $C$ be a hereditarily decomposable chainable continuum. Let $A$ be an end layer of $C$. Let $a$ and $b$ be two points of $C$ with $a \in A$ and $b \notin A$ and let $g:[a, b] \rightarrow C$ be a continuous mapping such that $g(a) \notin A$ and $g(b) \in A$. Then there is $c \in[a, b]-A$ such that $g(c)=c$.

Proof of Lemma 2.5. Since $A$ is an end layer of $C$, there is a continuum $U \subset C$ such that $b \notin U, g(a) \notin U, A \subset \operatorname{Int}(U)$ and $C-U$ is connected. Since $A$ is nowhere dense there is a point $u \in U-A$ so close to $a$ that $g(u) \notin U$. Suppose that $g$ does not have a fixed point in $[u, b]$. Then there is $\varepsilon>0$ such that for every $z \in[u, b]$ we have $d(z, f(z))>\varepsilon$, where $d$ denotes the distance function. Let $\delta$ be the minimum of $\varepsilon, d(A, C-U), d(b, U)$ and $d(g(u), U)$. Since $C$ is chainable, there is a $\delta$-map $h: C \rightarrow[0,1]$. Observe that if for some $t, h^{-1}(t)$ intersects $A$, then $h^{-1}(t)$ is contained in $U$. Since $h(C-U)$ is connected, it cannot contain both 0 and 1 . Without loss of generality we can assume that $h^{-1}(0) \subset U$. Define $F$ to be the set $\{z \in[u, b] \mid h(z)>h(g(z))\}$. Observe that $F$ is open, closed, $u \notin F$ and $b \in F$. This contradicts the connectivity of $[u, b]$. 
Lemma 2.6. Let $X$ be a hereditarily decomposable chainable continuum. Let $a, b, c$ and $d$ be points of $X$. Let $A$ and $B$ denote the layers of $[a, b]$ containing $a$ and $b$, respectively. Let $C$ and $D$ denote the layers of $[c, d]$ containing $c$ and $d$, respectively. Suppose that $f$ is a mapping of the irreducible continuum containing $a, b, c$ and $d$ into $X$ such that $f(a)=c, f(b)=d$, $f(d) \in A$ and $b \in(a, f(c)]$. Assume also that either $[a, b] \cap[c, d] \subset B-C$ or $[a, b] \cap[c, d] \subset B-D$. Then $f$ has a periodic point of period 2 .

Proof of Lemma 2.6. We will start by proving the following claim.

Claim 2.6.1. We can assume that $f([a, b])=[c, d]$.

Proof of Claim 2.6.1. Suppose that there is a point $q \in[a, b)$ such that $f(q)=$ $d$. Since $[a, q]$ and $[c, d]$ are disjoint, the diagram $[a, q] \rightleftarrows[c, d]$ implies the existence of a point of period 2. So we can assume that no point of $[a, b)$ is mapped by $f$ onto $d$.

Let $D^{\prime}$ be the layer of $d$ in $f([a, b]) . D^{\prime}$ is an end layer of $f([a, b])$ (see Lemma 2.2) and $D^{\prime}$ and the image of $[a, b)$ are disjoint (see Lemma 2.3). In particular $f(a) \notin D^{\prime}$. Hence $D^{\prime}=D$.

Since $[a, b]$ is disjoint either with $C$ or with $D$, we can assume that $[a, b]$ is not contained in $f(C) \cap f(D)$, because otherwise a periodic point of period 2 could be easily obtained.

Next we will prove that no point of $(a, b)$ is mapped by $f$ onto $c$. Suppose, on the contrary, that there is a point $q \in(a, b)$ such that $f(q)=c$. Since $b \in(a, f(c)]$, we have that $q \in(a, f(c)]$. If $q$ were in the layer of $f(c)$ in $[a, f(c)]$ then $[a, q]=[a, f(c)]$ would contain $b$. This contradiction proves that $q \in(a, f(c))=(f(d), f(c))$. Since $[a, b]$ is not contained in $f(C) \cap f(D)$, it follows from Lemma 2.4 that there is a point $r \in(c, d)$ such that $f(r)=q$. Since $r \in(f(q), f(b))$, it follows by Lemma 2.4 that there is a point $t \in[q, b)$ such that $f(t)=r$. Observe that $[c, r] \subset f([q, t])$ and $[q, t] \subset f([c, r])$. Since $[q, t]$ and $[c, r]$ are disjoint, the diagram $[q, t] \rightleftarrows[c, r]$ implies the existence of a point of period 2. So we can assume that no point of $(a, b)$ is mapped by $f$ onto $c$. Let $C^{\prime}$ be the layer of $c$ in $f([a, b]) . C^{\prime}$ is an end layer of $f([a, b])$ (see Lemma 2.2) and $C^{\prime}$ and the image of $(a, b)$ are disjoint (see Lemma 2.3). It follows from Lemma 2.1 that $C^{\prime} \subset f(A)$. Since $[c, d]$ is not contained in $f(A)$, then $d \notin C^{\prime}$. Since $c$ and $d$ belong to different end layers of $f([a, b])$, we have that $f([a, b])=[c, d]$.

Observe that we can assume that $[a, b]$ is not contained in $f^{2}(Q)$, for any continuum $Q \subset[a, b)$. Otherwise $f^{2}$ would have a fixed point in $Q$, which would not be a fixed point for $f$, because $f([a, b])=[c, d]$ is disjoint with $[a, b)$. In particular, $[a, b]$ is not contained in $f^{2}(A)$.

Now we will prove that no point of $(a, b)$ is mapped by $f^{2}$ onto $b$. Suppose, on the contrary, that there is a point $q \in(a, b)$ such that $f^{2}(q)=b$. Denote by $Q$ the layer of $q$ in $[q, b]$. Since $f^{2}(b) \in A$ and $[a, b]$ is not contained in $f^{2}(Q)$, it follows from Lemma 2.4 that there is a point $t \in(q, b)$ such that 
$f^{2}(t)=q$. Since $[q, t]$ is contained in $f^{2}([q, t]), f^{2}$ has a fixed point in $[q, t]$. This point is a periodic point of period 2 , because $f([q, t]) \subset[c, d]$ is disjoint with $[q, t] \subset[a, b)$. So we can assume that no point of $(a, b)$ is mapped by $f^{2}$ onto $b$.

Using Lemma 2.4 we get that there is a point $r \in[c, d)$ such that $f(r)=b$. Using Lemma 2.4 again we get that there is a point $a^{\prime} \in[a, b)$ such that $f\left(a^{\prime}\right)=r$. Since $f^{2}\left(a^{\prime}\right)=b$, we have that $a^{\prime} \in A$.

Let $B^{\prime}$ be the layer of $b$ in $f^{2}([a, b]) . \quad B^{\prime}$ is an end layer of $f^{2}([a, b])$ (see Lemma 2.2) and $B^{\prime}$ and $f^{2}((a, b))$ are disjoint (see Lemma 2.3). Since $b \in f^{2}(A)$, it follows from Lemma 2.1 that $B^{\prime} \subset f^{2}(A)$. Since $[a, b]$ is not contained in $f^{2}(A)$, then $a \notin B^{\prime}$ and $B^{\prime}=B$. Now, it follows from Lemma 2.5 that $f^{2}$ has a fixed point in $[a, b)$. This point is a periodic point of period 2 for $f$.

\section{AN ORDER OF A HEREDITARILY DECOMPOSABLE CHAINABLE CONTINUUM}

Since our proof of the theorem follows that in the case of an interval, it is convenient to preserve as much of the language applying to the real line as possible. It is particularly convenient to order points of a hereditarily decomposable chainable continuum $X$ so that if $a<c<b$ then $c \in[a, b]$. Given a Kuratowski function $g$ defined on $X$ and two points $a$ and $b$ belonging to two different layers we can say that $a<b$ if $g(a)<g(b)$. If two points belong to the same layer we will have to use a Kuratowski function for this layer. Then considering layers of layers and again layers of layers, as in [9 and 7], may be necessary. The order obtained in this way will be by no means unique but it will not matter in the proof of the theorem.

Define $\mathbf{C}_{0}$ to be $\{X\}$. If $\alpha=\beta+1$ then $\mathbf{C}_{\alpha}$ will consist of points belonging to $\mathbf{C}_{\beta}$ and the layers of nondegenerate elements of $\mathbf{C}_{\beta}$. For a limit ordinal $\alpha$ define $\mathbf{C}_{\alpha}$ to be the coarsest decomposition of $X$ refining all $\mathbf{C}_{\beta}$ 's for $\beta<\alpha$. Observe that in the last case $\mathrm{C}_{\alpha}$ consists of the intersections $\bigcap_{\beta<\alpha} E_{\beta}$, where $E_{\beta} \in \mathbf{C}_{\beta}$.

For every $x \in X$ denote by $C_{\alpha}(x)$ the element of $\mathbf{C}_{\alpha}$ containing $x$. It was proven in [9] that $C_{\alpha}(x)$ is $\{x\}$ for some countable $\alpha$. It was shown in [7] that there is a countable $\tau$ so that $C_{\tau}(x)$ is $\{x\}$ for every $x \in X$.

For an ordinal number $\alpha$, a (not necessarily continuous) function $g_{\alpha}: X \rightarrow$ $[0,1]$ will be called an $\alpha$-function if $g_{\alpha}$ restricted to any nondegenerate $C \in \mathbf{C}_{\alpha}$ is a Kuratowski function. Consider a collection $\mathscr{G}=\left\{g_{\alpha}\right\}_{\alpha<\tau}$, where $g_{\alpha}$ is an $\alpha$-function.

If $\alpha$ is an ordinal number, $x \in X$ and $g_{\alpha}(x)=t$ denote by $\alpha_{0}(x)$ the connected set $g_{\alpha}^{-1}([0, t)) \cap C_{\alpha}(x)$ and by $\alpha_{1}(x)$ denote the connected set $g_{\alpha}^{-1}((t, 1]) \cap C_{\alpha}(x)$. For $i$ either 0 or 1 denote by $S_{i}(\alpha, x)$ the set $\bigcup_{\beta \leq \alpha} \beta_{i}(x)$.

We will construct a collection $\mathscr{G}$ in such a way that the set $S_{i}(\alpha, x) \cap C_{\beta}(x)$ is connected for every $\beta<\alpha \leq \tau, x \in X$ and $i=0,1$. 
Let $g_{0}$ be a Kuratowski function for $X$. Suppose that for every ordinal $\beta$ less than a certain ordinal $\alpha$, a $\beta$-function has been constructed in such a way that $S_{i}(\delta, x) \cap C_{\beta}(x)$ is connected for every $\beta<\delta<\alpha, x \in X$ and $i=0,1$. It is sufficient to extend the collection by constructing $g_{\alpha}$. To do that we will define $g_{\alpha}$ on every $C \in \mathbf{C}_{\alpha}$.

If $\alpha=\beta+1$ then for some $x \in C$ consider the sets $\operatorname{cl}\left(\beta_{0}(x)\right) \cap C$ and $\operatorname{cl}\left(\beta_{1}(x)\right) \cap C$ and denote them $S_{0}(C)$ and $S_{1}(C)$, respectively. Observe that the sets do not depend on the choice of a point $x$ within $C$. Since $C$ is a layer of the element $D$ of $\mathbf{C}_{\beta}$ containing it, we have that $C=S_{0}(C) \cup S_{1}(C)$. Observe also that the sets do not separate $C$ because otherwise $D$ would contain a triod. Now define $g_{\alpha}$ on $C$ as a Kuratowski function such that $0 \in g_{\alpha}\left(S_{0}(C)\right)$ and $1 \in g_{\alpha}\left(S_{1}(C)\right)$. It is easy to see that $g_{\alpha}$ has the required properties.

Suppose that $\alpha$ is a limit ordinal. Let $i$ be either 0 or 1 . Observe that $S_{i}(\delta, x) \cap C_{\beta}(x)$ does not depend on the choice of a point $x$ within $C$. Denote by $S_{i}(\beta, C)$ the set $\operatorname{cl}\left(C_{\beta}(x) \cap \bigcup_{\delta<\alpha} S_{i}(\delta, x)\right)$. Since $S_{i}(\gamma, x) \subset S_{i}(\delta, x)$ for $\gamma \leq \delta<\alpha, S_{i}(\beta, C)$ is connected. Since $C$ is contained in a layer of the element $D$ of $\mathbf{C}_{\beta}$ containing it, we have that $C \subset S_{0}(\beta, C) \cup S_{1}(\beta, C)$. Observe also that the sets $C-S_{0}(\beta, C)$ and $C-S_{1}(\beta, C)$ do not separate $C$ because otherwise $D$ would contain a triod. Let $S_{i}(C)$ denote $\bigcap_{\beta<\alpha} S_{i}(\beta, C)$. Observe that $S_{i}(C)$ does not separate $C$ and $C=S_{0}(C) \cup S_{1}(C)$. Now define $g_{\alpha}$ on $C$ as a Kuratowski function such that $0 \in g_{\alpha}\left(S_{0}(C)\right)$ and $1 \in g_{\alpha}\left(S_{1}(C)\right)$. It is easy to see that $g_{\alpha}$ has the required properties.

Let $a$ and $b$ be two points of $X$. Let $\alpha$ be the first ordinal number so that $g_{\alpha}(a) \neq g_{\alpha}(b)$. If $g_{\alpha}(a)<g_{\alpha}(b)$ then we will say that $a$ is less than $b$ or $a<b$, otherwise we will say that $a$ is greater than $b$ or $a>b$. If $a$ is less than each element of a set $S$, we will say that $a$ is to the left of $S$. If $a$ is greater than each element of a set $S$, we will say that $a$ is to the right of $S$. (Observe that $\mathscr{G}$ is not unique and different choice of $g_{\alpha}$ 's may yield a different order on $X$.)

The following proposition can be easily inferred from the construction and its proof will be omitted.

Proposition 3.1. If $a<c<b$ then $c \in[a, b]$.

\section{Auxiliary lemmas}

In this section we shall prove five lemmas to be used in the proof of the theorem. Let $X, Y$ and $f$ be as in Theorem 1.1. Let $x$ be a periodic point of period $n$. In view of the nature of the Sarkovskii ordering we shall assume that if $n$ is an odd number, then it is the least possible odd (nontrivial) period of $f$. If $n$ is even we shall assume that no odd $n$ could be chosen. Denote by $\operatorname{orb}(x)$ the orbit of $x$. Let $x_{1}, x_{2}, \ldots, x_{n}$ be $\operatorname{orb}(x)$ enumerated in increasing order. Since orb $(x) \subset X$ and $X$ is a continuum, the irreducible continuum containing orb $(x)$ is contained in $X$. Without loss of generality we can assume that $X$ is the irreducible continuum containing orb $(x)$. 
Lemma 4.1. If a continuum $I \subset X$ contains at least two points of the orbit of $x$ and $I \subset f(I)$, then $X \subset f^{n-2}(I)$. Moreover, if $f^{n-3}(I)$ does not contain $X$, then $\operatorname{orb}(x)$ can represented as a sequence $y_{1}, y_{2}, \ldots, y_{n}$ such that

(a) $y_{1}=f\left(y_{n}\right)$ and $y_{j+1}=f\left(y_{j}\right)$ for $j=1,2, \ldots, n-1$, and

(b) $y_{1}, y_{2} \in I$ and $y_{j+2} \in f^{j}(I)-f^{j-1}(I)$ for $j=1,2, \ldots, n-2$.

Proof of Lemma 4.1. Observe that $f^{j}(I) \subset f^{j+1}(I)$ for every $j \geq 0$. If $f^{j}(I)$ does not contain orb $(x)$, then $f^{j+1}(I)$ contains more points of the orbit than $f^{j}(I)$. Since we are starting with at least two points of the orbit in $I$, we have to pick up the rest in at most $n-2$ iterations of $f$. If $n-2$ iterations are needed, then there were exactly two points of the orbit in $I$ and with every iteration exactly one point is obtained.

Definition 4.2. Let $I \subset X$ be a continuum with end layers $I_{0}$ and $I_{1}$. We will say that $I$ is central with respect to $\operatorname{orb}(x)$ if the following conditions are satisfied:

(*) there are points $x_{i} \in I_{0}$ and $x_{j} \in I_{1}$ such that

$x_{i}<x_{j}$,

$f\left(x_{j}\right)$ is either in $I_{0}$ or to the left of it and

$f\left(x_{i}\right)$ is either in $I_{1}$ or to the right of it,

(**) $I-\left(I_{0} \cup I_{1}\right)$ does not contain points of $\operatorname{orb}(x)$ and

$(* * *) I$ is a maximal continuum satisfying $(*)$ and $(* *)$, and, if possible, $I$ is not contained in one layer of $X$.

Lemma 4.3. There is a continuum I central with respect $\operatorname{orb}(x)$.

Proof of Lemma 4.3. To prove the lemma it is enough to show that there is a continuum $I$ satisfying conditions $(*)$ and $(* *)$. Let $i$ be the last index for which $f\left(x_{i}\right)>x_{i}$. Then $f\left(x_{i+1}\right) \leq x_{i}<x_{i+1} \leq f\left(x_{i}\right)$ and $I$ can be defined as $\left[x_{i}, x_{i+1}\right]$.

Lemma 4.4. Let I be a continuum irreducible between two points of $\operatorname{orb}(x)$ such that $I \subset f(I)$. Let $z$ be a point of $\operatorname{orb}(x)$ such that $I$ is contained in the layer of $z$ in $[z, f(z)]$. Then $I$ is not central with respect to $\operatorname{orb}(x)$.

Proof of Lemma 4.4. Without loss of generality we can assume that $f(z)$ is to the right of $z$. Denote by $L$ the layer of $[z, f(z)]$ containing $z$ and $I$. Let $x_{k}$ be the greatest point of the orbit of $x$ belonging to $L$. Let $j$ be the least index greater than $k$ such that $f\left(x_{j}\right)$ is either in $L$ or to the left of it. Denote by $I^{\prime}$ the continuum $\left[x_{j-1}, x_{j}\right]$. Let $I_{0}^{\prime}$ denote the layer of $I^{\prime}$ containing $x_{j-1}$ and let $I_{1}^{\prime}$ denote the other end layer of $I^{\prime}$.

Claim 4.4.1. We can assume that $I^{\prime} \cap L=\varnothing$.

Proof of the claim. Suppose that $I^{\prime} \cap L \neq \varnothing$. Since $L$ is the left layer of [z,f(z)], and $I^{\prime}$ intersects $L$ and contains a point to the right of $L$, we have that $L$ is contained in one layer of $I^{\prime}$. Since $I_{0}^{\prime}$ is the left end layer of $I^{\prime}$ and 
$x_{j-1}$ is either in $L$ or to the right of $L, L \subset I_{0}^{\prime}$. Then either $j=k+1$ and $f(z) \geq x_{j}$ or $j>k+1$. In the last case it follows from the choice of $j$ that one of the points $x_{k+1}, \ldots, x_{j-1}$ is mapped by $f$ either onto $x_{j}$ or to the right of it. So in any case $I_{0}^{\prime}$ contains a point of the orbit of $x$ which is mapped by $f$ either into $I_{1}^{\prime}$ or to the right of it. Now, $I^{\prime}$ satisfies the conditions $(*)$ and (**) of Definition 4.2. Since $I$ is contained in $I^{\prime}, I$ cannot be central.

Let $q$ be the least positive integer such that $I^{\prime} \subset f^{q}(I)$. By Lemma 4.1, $q \leq n-2$. Since $f\left(x_{j-1}\right)$ is to the right of $L$ and $f\left(x_{j}\right)$ is either in $L$ or to the left of it, we have that $L \subset f\left(I^{\prime}\right)$ and $I \subset L \subset f\left(I^{\prime}\right)$. Since $I$ an $I^{\prime}$ are disjoint, the diagram $f \subset I \underset{f}{\stackrel{f^{q}}{\rightleftarrows}} I^{\prime}$ implies the existence of a periodic point of an odd period less or equal to $q+2$. This cannot happen in the case of $n$ even because otherwise an odd $n$ would be chosen. If $n$ is odd, the choice of $n$ as the least odd period implies $q=n-2$.

Claim 4.4.2. $f\left(x_{j-1}\right)=x_{j}$.

Proof of Claim 4.4.2. Let $x_{t}$ be the point of the orbit of $x$ such that $f\left(x_{t}\right)=x_{j}$. It follows from Lemma 4.1 that $x_{t} \in f^{n-3}(I)-f^{n-4}(I)$. Now, either $x_{t}=x_{j-1}$ or $x_{t}>x_{j-1}$. In that last case $x_{t}$ would be to the right of $x_{j}$ and $x_{j}$ would be contained in $f^{n-3}(I)$.

To finish the proof of the lemma set $i=j-1$. Observe that conditions $(*)$ and $(* *)$ of Definition 1 are satisfied. Since $X \subset I^{\prime} \cup f^{n-3}(I), x_{j} \notin f^{n-3}(I)$ and $f^{n-3}(I)$ is closed, we have that $I^{\prime}$ is not contained in one layer of $X$. Therefore $I$, which is contained in one layer of $X$, cannot be central.

Lemma 4.5. Let $I$ be a continuum central with respect to $\operatorname{orb}(x)$. Let $I_{0}$ be the left end layer of $I$ and let $I_{1}$ be the right end layer. Suppose that there is a point of $I_{0}$ which is sent by $f$ to $I_{0}$ or to the left of $i$. Then $X$ contains periodic points of all periods. (Also the same is true if $I_{1}$ contains a point that is sent by $f$ to $I_{1}$ or to the right of it.)

Proof of Lemma 4.5. Let $x_{i}$ and $x_{j}$ satisfy the conditions of Definition 4.2. It follows that $I \subset f\left(I_{0}\right)$. Suppose that there is a point $t \in I-I_{0}$ such that $I_{0} \subset f\left(\left[t, x_{j}\right]\right)$. Since $I_{0}$ and $\left[t, x_{j}\right]$ are disjoint the diagram $\subset I_{0} \rightleftarrows\left[t, x_{j}\right]$ yields periodic points of all periods. So we can assume that there is no $t \in I-I_{0}$ such that $I_{0} \subset f\left(\left[t, x_{j}\right]\right)$.

Let $F_{0}$ and $F_{1}$ be the end layers of $f(I)$. First we will prove that $I$ is contained either in $F_{0}$ or in $F_{1}$. Suppose that $I$ intersects an interior layer of $f(I)$. Then, since $I-I_{0}$ is dense in $I$ there is a point $q$ which belongs to both $I-I_{0}$ and $f(I)-\left(F_{0} \cup F_{1}\right)$. By Lemma 2.2 there is a point $t \in I-I_{0}$ such that $f(t)=q$. Since $I_{0}$ is the left end layer of $I, f\left(x_{j}\right)$ is either in $I_{0}$ or to the left of it, and $q$ is to its right, we have that $I_{0} \subset f\left(\left[t, x_{j}\right]\right)$. Thus we can assume that $I \subset F_{1}$. If $f\left(x_{j}\right)$ also belongs to $F_{1}$, then it follows from 
Lemma 2.3 that there is a point $t \in I-I_{0}$ such that $F_{1} \subset f\left(\left[t, x_{j}\right]\right)$. Since $I_{0} \subset I \subset F_{1} \subset f\left(\left[t, x_{j}\right]\right)$, we can assume that $f\left(x_{j}\right) \notin F_{1}$. Since $x_{j} \in F_{1}$, we have that $F_{1}$ is an end layer of $\left[f\left(x_{j}\right), x_{j}\right]$. Now, Lemma 4.4 contradicts the choice of $I$ as a continuum central with respect to $\operatorname{orb}(x)$.

Lemma 4.6. Let $I=\left[x_{i}, x_{j}\right]$ be a continuum central with respect to $\operatorname{orb}(x)$. Let $J \subset X$ be a continuum and let $k \geq 1$ be an integer such that

(i) $J=[y, z], y$ and $z$ are points of $\operatorname{orb}(x), y, z$ and $f(z)$ are on the same side of $\left(x_{i}, x_{j}\right)$, but $f(y)$ is on the other side and

(ii) $J \subset f^{k}(I)$.

Then $X$ contains a periodic point of odd period $m$ with $1<m \leq k+2$.

Proof of Lemma 4.6. Assume that $x_{i}, x_{j}, I_{0}$ and $I_{1}$ satisfy the conditions of Definition 4.2. Let us assume further that $J$ contains as few elements of orb $(x)$ as possible while satisfying (i) and (ii).

Claim 4.6.1. We can assume that $I \cap J$ contains a point $p$ fixed under $f$, and in particular $I \cap J \neq \varnothing$.

Proof of Claim 4.6.1. The diagram $f \subset I \underset{f}{\stackrel{f^{k}}{\rightleftarrows}} J$ implies that for every $s \geq k+1$ there is a point $p \in I$ such that $f^{s}(p)=p$ and $f^{s-1}(p) \in J$. Set $s$ to be either $k+1$ or $k+2$ whichever is odd. Then $p$ is a periodic point of an odd period $m \leq s \leq k+2$. So either the lemma is true or $m=1$ and consequently $p$ is a fixed point of $f$.

Using Lemma 4.5, one can easily infer that $J-I \neq \varnothing$. Without loss of generality we can assume that $y$ and $z$ are on the same side of $\left(x_{i}, x_{j}\right)$ as $x_{j}$. Let $J_{0}$ be the layer of $J$ containing $p . J_{0}$ is an end layer of $J$. Let $J_{1}$ be the other end layer of $J$. Since, by Lemma 4.5, $p \notin I_{1}$ and $J_{0}$ contains a point to the right of $\left(x_{i}, x_{j}\right)$ and $I_{1}$ is an end layer of $I$, we have that $I_{1} \subset J_{0}$.

Claim 4.6.2. $J-\left(J_{0} \cup J_{1}\right)$ does not contain points of $\operatorname{orb}(x)$.

Proof of Claim 4.6.2. For, if so, $y$ or $z$ could be replaced by this point giving a new $J$ with fewer points of $\operatorname{orb}(x)$.

Observe that $z \in J_{1}$, because otherwise $z \in J_{0}$ and $y$ could be replaced by $x_{j}$ yielding a $J$ with fewer points of $\operatorname{orb}(x)$.

Claim 4.6.3. We can assume that no point in $J$ is sent by $f$ to the left of both $I$ and $J$.

Proof of Claim 4.6.3. First we will prove that $I_{0}$ is contained in an end layer of $f(J)$. Suppose that some point $q \in I_{0}$ is in an interior layer of $f(J)$. By Lemma 2.2 there is a point $t \in J-J_{0}$ so that $f(t)=q$. Observe that $f([t, z])$ contains $q \in I_{0}$ and $f(z)$ which is to the right of $\left(x_{i}, x_{j}\right)$. Thus $I \subset f([t, z])$. Since $[t, z] \subset J-J_{0}$, then $[t, z]$ and $I$ are disjoint and the 
diagram $f \subset I \underset{f}{\stackrel{f^{k}}{\rightleftarrows}}[t, z]$ implies that there is a periodic point of an odd period $m$, where $1<m \leq k+2$. Therefore we can assume that $I_{0}$ is contained in an end layer of $f(J)$. Denote this layer by $F$.

We can assume that $f(z) \notin F$. Otherwise, there is a point $t \in J-J_{0}$ such that $F \subset f([t, z])$ (see Lemma 2.3). It follows that $x_{i} \in I_{0}$ is in $f([t, z])$. Since $f(z)$ and $x_{i}$ are on opposite sides of $\left(x_{i}, x_{j}\right), I \subset f([t, z])$. Now we have $I$ and $[t, z]$ disjoint and the standard argument with the diagram $f \subset I \underset{f}{\stackrel{f^{k}}{\rightleftarrows}}[t, z]$ applies.

Now suppose Claim 4.6.3 is false. Then there is a point $c \in f(J)$ which is to the left of $J$ and $I$. Since $f(z) \notin F, f(z)$ is to the right of $I_{0} \subset F$ and $c$ is to the left, we have that $c \in F$.

Since $F$ is an end layer of $f(J)$ and $f(z) \notin F, F$ is an end layer of $[d, f(z)]$ for every point $d \in F$. Observe that the continuum $I \cup J$ intersects $F$ and does not contain $c$. So $f(z) \notin I \cup J$. Since $z$ and $f(z)$ are on the same side of $\left(x_{i}, x_{j}\right), f(z)$ is to the right of $z$ and $I \cup J \subset f(J)$. Again since $I \cup J$ is a continuum meeting $F$ but not containing $F$, we have that $I \cup J \subset F$. In particular $z \in F$. Since $f(z) \notin F, F$ is a layer of $[z, f(z)]$. Now, Lemma 4.4 contradicts the choice of $I$ as a central continuum.

Claim 4.6.4. We can assume that $I-J \neq \varnothing$.

Proof of Claim 4.6.4. Suppose that $I \subset J$. Then $I \subset J_{0}$. By Claim 4.6.3, there is a point $u \in \operatorname{orb}(x) \cap J_{0}$ such that $f(u)$ is to the right of $J_{0}$. Since $J_{0}$ is the first layer of $J$, we have that $J_{0}$ is contained in the layer of $u$ in $[u, f(u)]$. Again, Lemma 4.4 contradicts the choice of $I$ as a central continuum.

Claim 4.6.5. There is a point $x_{s} \in I_{0}$ such that $f\left(x_{s}\right)$ is to the right of $J_{0}$.

Proof of Claim 4.6.5. Observe that there is no point $u \in \operatorname{orb}(x) \cap J_{0}$ so that $f(u)$ is to the right of $\left(x_{i}, x_{j}\right)$, since otherwise $J$ could be replaced by $\left[x_{j}, u\right]$ which would contain fewer points of $\operatorname{orb}(x)$.

Suppose the claim is not true. Then by Claim 4.6.3 and Lemma 4.5 all points of the orbit contained in $I_{0} \cup J_{0}$ would be sent by $f$ to $I_{0} \cup J_{0}$. So the orbit of $x$ would be contained in $I_{0} \cup J_{0}$ which is not the case.

Since $f\left(x_{j}\right)$ is to the left of $\left(x_{i}, x_{j}\right)$ and $p$ is a fixed point not in $I_{0}$, there is a point $q \in\left[x_{j}, p\right]$ such that $f(q)=x_{s}$.

Claim 4.6.6. There is a point $r \in J-J_{0}$ such that $f(r)=q$.

Proof of Claim 4.6.6. Suppose that the claim is false. Since $J-J_{0}$ is dense in $J$, by Lemma 2.2 we can assume that $q$ belongs to one of the end layers of $f(J)$. Denote this layer by $F$. If $f(z) \in F$, then it follows from Lemma 2.3 that there is a point $t \in J-J_{0}$ so that $F \subset f([t, z])$ and the claim is true. Thus we can assume that $f(z) \notin F$. Since $f(z)$ is to the right of $\left(x_{i}, x_{j}\right)$, 
$I_{0}$ is to the left of $\left(x_{i}, x_{j}\right)$ and $I_{0} \subset f(J)$, we have that $I_{0} \subset F$. Let $v$ be a point of $f(J)-F$. Observe that $F \subset[q, v]$, so if $v \in J$, then $I_{0}$ would be contained in $J$, which contradicts Claim 4.6.4. Therefore $f(z)$ is to the right of $J$ and $J$ is contained in $F$. Since $I$ and $z$ are contained in $F$ which is an end layer of $[z, f(z)]$ we can use Lemma 4.4 and get a contradiction with the choice of $I$ as a central continuum.

Claim 4.6.7. There is a point $t \in J-J_{0}$ such that $I \subset f^{2}([t, r])$.

Proof of Claim 4.6.7. Let $v \in J-\left(J_{0} \cap J_{1}\right)$. Observe that by Claims 4.6.2 and 4.6.5 $v \in\left[x_{s}, f\left(x_{s}\right)\right]$. Since $J$ is not contained in $\left[x_{s}, v\right]=I \cup[y, v]$, we have that $v$ is not in the layer of $f\left(x_{s}\right)$ in $\left[x_{s}, f\left(x_{s}\right)\right]$. Since $f^{2}(r)=x_{s}$ and $f^{2}(q)=f\left(x_{s}\right)$, using Lemma 2.4(i) with $a=r, b=q, c=v$ and $f=f^{2}$, we get an element $t$ in $[q, r]-J_{0}$ so that $f^{2}(t)=v$. Observe that $I \subset f^{2}([t, r])$.

Since $[t, r]$ and $I$ are disjoint the diagram $f \subset I \underset{f^{2}}{\stackrel{f}{\rightleftarrows}}[t, r]$ implies the existence of points of all periods (greater than 2), which completes the proof of Lemma 4.6.

\section{Proof OF THeOREM 1.1}

Case 1: $n=3$.

Let $I=\left[x_{i}, x_{j}\right]$ be a continuum central with respect to orb $(x)$. (See Lemma 4.3.) Observe that $I$ is not equal $\left[x_{1}, x_{3}\right]$, because otherwise Lemma 4.5 could be used to reach the desired conclusion. Since the cases $I=\left[x_{1}, x_{2}\right]$ and $I=\left[x_{2}, x_{3}\right]$ are symmetric, we can assume without loss of generality that $I=$ $\left[x_{2}, x_{3}\right]$. Since $f\left(x_{2}\right)>x_{2}, f\left(x_{2}\right)=x_{3}$. Since $f$ does not switch $x_{2}$ and $x_{3}, f\left(x_{3}\right)=x_{1}$ and $f\left(x_{1}\right)=x_{2}$. We can assume that $x_{1} \notin\left[x_{2}, x_{3}\right]$, because otherwise $x_{1}$ would belong to the layer of $x_{2}$ in $\left[x_{2}, x_{3}\right]$ and Lemma 4.5 could be applied. We can also assume that $x_{3} \notin\left[x_{1}, x_{2}\right]$, because otherwise a contradiction follows from Lemma 4.4 applied with $z=x_{3}$. Let $L$ denote the layer of $x_{2}$ in $\left[x_{1}, x_{3}\right]$. Since neither one of the continua $\left[x_{1}, x_{2}\right]$ and $\left[x_{2}, x_{3}\right]$ contains the other, $L$ is not an end layer of $\left[x_{1}, x_{3}\right]$. Denote by $W$ and $Z$ the closures of $\left[x_{1}, x_{2}\right]-L$ and $\left[x_{2}, x_{3}\right]-L$, respectively. Since $L$ is nowhere dense, $\left[x_{1}, x_{3}\right]=W \cup Z$. We will now consider two cases $x_{2} \in W$ and $x_{2} \in Z$. Case 1.1: $x_{2} \in W$. Observe that in this case $W=\left[x_{1}, x_{2}\right]$ and $\left[x_{1}, x_{2}\right)$ does not intersect $\left[x_{2}, x_{3}\right]$. Observe that $f^{2}\left(x_{1}\right)=x_{3}$ and $f^{2}\left(x_{2}\right)=x_{1}$. Using Lemma 2.4 one can get a point $t \in\left[x_{1}, x_{2}\right)$ such that $f^{2}(t)=x_{2}$. Since $\left[x_{1}, t\right]$ and $\left[x_{2}, x_{3}\right]$ are disjoint, the diagram $\left[x_{1}, t\right] \underset{f}{\stackrel{f^{2}}{\rightleftarrows}}\left[x_{2}, x_{3}\right]$ ) implies the existence of periodic points of all periods greater than 2 . To prove that $f$ has a periodic point of period 2 set $a=x_{1}, b=c=x_{2}, d=x_{3}$ and then use Lemma 2.6.

Case 1.2: $x_{2} \in Z$. In this case $Z=\left[x_{2}, x_{3}\right]$ and $\left(x_{2}, x_{3}\right]$ does not intersect $\left[x_{1}, x_{2}\right]$. To prove that $f$ has a periodic point of period 2 , take $b \in\left[x_{2}, x_{3}\right]$ 
such that $f(b)=x_{2}, a=x_{3}, c=x_{1}, d=x_{2}$ and then use Lemma 2.6. Now we will prove that $f$ has a periodic point of period 4 . Observe that $x$ is a periodic point of period 3 for $f^{2}$. Since we already proved that the existence of such point implies (in both cases) that $f^{2}$ has a periodic point of period 2 . Obviously this point has period 4 under $f$.

Denote by $I_{1}$ the layer of $x_{3}$ in $\left[x_{2}, x_{3}\right]$. By Lemma 4.5 we can assume that $x_{3} \notin f\left(I_{1}\right)$. Using Lemma 2.4 (ii) one can get that there is a point $q \in\left(x_{2}, x_{3}\right)$ such that $f(q)=x_{2}$.

Denote by $Q$ the layer of $q$ in $\left[x_{2}, q\right]$. Suppose that $x_{3} \in f(Q)$. Then $Q \subset f(Q)$ and $\left[x_{1}, x_{2}\right] \subset f^{2}(Q)$. Since $\left[x_{1}, x_{2}\right]$ and $Q$ are disjoint, the diagram $\left[x_{1}, x_{2}\right] \underset{f^{2}}{\stackrel{f}{\rightleftarrows}} Q \supset$ implies the existence of periodic points of all periods greater than 2. Thus we can assume that $x_{3} \notin f(Q)$. Again, using Lemma 2.4(ii) one can get that there is a point $t \in\left(x_{2}, q\right)$ such that $f(t)=q$.

Observe that $[t, q] \subset f([t, q]), f^{3}(t)=f^{2}(q)=f\left(x_{2}\right)=x_{3}$ and $f^{3}(q)=$ $f\left(x_{3}\right)=x_{1}$. It follows that $\left[x_{1}, x_{2}\right] \subset f^{3}([t, q])$. Let $m$ be any integer greater than 4. The diagram $\left[x_{1}, x_{2}\right] \underset{f^{3}}{\stackrel{f}{\rightleftarrows}}[t, q] \supset$ implies that there is a point $z \in$ $\left[x_{1}, x_{2}\right]$ such that $f^{m}(z)=z$ and $f^{k}(z) \in[t, q]$ for $1 \leq k \leq m-3$. Since $\left[x_{1}, x_{2}\right]$ and $[t, q]$ are disjoint, we have that $f^{k}(z) \neq z$ for $1 \leq k \leq m-3$. Then either $z$ is a periodic point of period $m$ and the construction is completed or $f^{m-2}(z)=z$ or $f^{m-1}(z)=z$. If $f^{m-1}(z)=z$, then $f(z)=f\left(f^{m-2}(z)\right)=$ $z$, which is a contradiction. If $f^{m-2}(z)=z$, then $f^{2}(z)=f^{2}\left(f^{m-2}(z)\right)=z$, so either $2=m-2$ or $2=m-1$, which is a contradiction.

Case 2: $n$ is an odd number greater than 3 .

Let $I=\left[x_{i}, x_{j}\right]$ be a continuum central with respect to $\operatorname{orb}(x)$ and let $I_{0}$ and $I_{1}$ be the left and right end layers of $I$. (See Lemma 4.3.) Let $m$ be the number of points of the orbit of $x$ on the same side of $\left(x_{i}, x_{j}\right)$ as $x_{i}$. Since $n$ is an odd number, then $m \neq n-m$ and there is a point $x_{s}$ such that $x_{s}$ and $f\left(x_{s}\right)$ are on the same side of $\left(x_{i}, x_{j}\right)$. Without loss of generality we can assume that $x_{s}$ is on the same side of $\left(x_{i}, x_{j}\right)$ as $x_{i}$. The continuum $J=\left[x_{s}, x_{i}\right]$ satisfies the assumptions of Lemma 4.6. By the first part of Lemma 4.1, $J \subset f^{n-2}(I)$. Since $n$ was chosen as the smallest nontrivial odd period of $f$, Lemma 4.6 implies that $J$ is not contained in $f^{n-3}(I)$ and therefore the second part of Lemma 4.1 applies. Let $y_{1}, y_{2}, \ldots, y_{n}$ be as in Lemma 4.1. Without loss of generality we can assume that $y_{1}<y_{2}$.

Proposition 5.1. $y_{n}<y_{n-2}<\cdots<y_{3}<y_{1}<y_{2}<y_{4}<\cdots<y_{n-3}<y_{n-1}$.

Proof of Proposition 5.1. Let $q$ be the last even number such that $y_{q-1}<y_{q-3}<$ $\cdots<y_{3}<y_{1}<y_{2}<y_{4}<\cdots<y_{q-2}<y_{q}$. By our assumption $q$ is at least 2. If $q=2$, then by the choice of $I$ and Lemma 4.1(b), we have $y_{3}<y_{1}$. Again by Lemma 4.1(b) either $y_{2}<y_{4}$ or $y_{4}<y_{3}$. If $y_{4}<y_{3}$ then $\left[y_{3}, y_{1}\right]$ 
would be a $J$ which by Lemma 4.6 would yield a point of period 3. In the case of $q>2$, if $y_{q}<y_{q+1}$, then $J=\left[y_{q-2}, y_{q}\right]$ would satisfy the assumptions of Lemma 4.6 with $k=q-2<n-2$, and the lemma would contradict the choice of $n$ as the least nontrivial odd period. Thus $y_{q+1}<y_{q}$, but since $y_{q+1}$ does not belong to $f^{q-2}(I)$ and $\left[y_{q-1}, y_{q}\right] \subset f^{q-2}(I)$, we have that $y_{q+1}<y_{q-1}$. Now, if $q+1<n$, then one can prove, repeating the argument, that $y_{q}<y_{q+2}$. This would contradict the choice of $q$.

Proposition 5.2. $\left[y_{n-4}, y_{n-1}\right]$ and $\left[y_{n}, y_{n-2}\right)$ are disjoint.

Proof of Proposition 5.2. First we will prove that $\left[y_{2}, y_{n-1}\right]$ and $\left[y_{n}, y_{n-2}\right)$ are disjoint. Suppose that this is not true: Then it follows that $y_{n-2} \in\left[y_{2}, y_{n-1}\right]$ and $\left[y_{n-2}, y_{2}\right]$ is contained in the layer of $y_{2}$ in $\left[y_{2}, y_{n-1}\right]$. Since $f\left(y_{n-2}\right)=$ $y_{n-1}$, Lemma 4.4 contradicts the choice of $I=\left[y_{1}, y_{2}\right]$ as a central continuum.

To complete the proof of the proposition it is enough to show that $\left[y_{n-4}, y_{2}\right]$ and $\left[y_{n}, y_{n-2}\right)$ are disjoint. Suppose that this is not true. Then it follows that $y_{n-2} \in\left[y_{n-4}, y_{2}\right] \subset f^{n-5}(I)$ and $J=\left[y_{n}, y_{n-2}\right] \subset f^{n-3}(I)$. In this case Lemma 4.6 contradicts the choice of $n$.

$A$ construction of a periodic point of period 2. Set $a=y_{n}, b=y_{n-2}, c=y_{1}$ and $d=y_{n-1}$. Let $B$ denote the layer of $y_{n-2}$ in $\left[y_{n}, y_{n-2}\right]$. Let $D$ denote the layer of $y_{n-1}$ in $\left[y_{1}, y_{n-1}\right]$. It follows by Lemma 4.4 and the choice of $I$ that $y_{n-1} \notin\left[y_{n}, y_{n-2}\right]$. Thus $D$ does not intersect $\left[y_{n}, y_{n-2}\right]=[a, b]$. It follows from Proposition 2.2 that $\left[y_{n}, y_{n-2}\right] \cap\left[y_{1}, y_{n-1}\right]=[a, b] \cap[c, d] \subset B$. Now, Lemma 2.6 can be used to get a periodic point of period 2 .

A construction of a periodic point of period 4. Observe that $x$ is a periodic point of period $n$ for $f^{2}$. So $f^{2}$ has periodic points of nontrivial odd periods. Using either the previous construction or the one for $n=3$ we get that $f^{2}$ has a periodic point of period 2. Obviously the point has period 4 under $f$.

A construction of periodic points for periods equal to or greater than $n-1$. Since $y_{n-1} \notin f^{n-4}(I), y_{1}, y_{2} \in f^{n-4}(I), f\left(y_{n-2}\right)=y_{n-1}$ and $f\left(y_{n}\right)=y_{1}$, it follows from Lemma 2.4 that there is a point $q \in\left[y_{n}, y_{n-2}\right)$ such that $f(q)=y_{2}$. (Note that for this argument $n$ has to be greater than 3.) Let $m$ be an integer equal or greater than $n-1$. The diagram

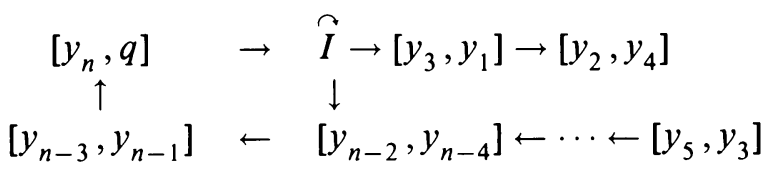

implies that there is a point $z \in\left[y_{n}, q\right]$ such that $f^{m}(z)=z, f^{m-1}(z) \in$ $\left[y_{n-3}, y_{n-1}\right], f^{m-2}(z) \in\left[y_{n-2}, y_{n-4}\right], \ldots, f^{m-n+3}(z) \in\left[y_{3}, y_{1}\right]$ and $f^{k}(z) \in I$ for $1 \leq k \leq m-n+2$. By Proposition 5.2 we have that $f^{m-1}(z) \neq z$ and $f^{k}(z) \neq z$ for $1 \leq k \leq m-3$. Then either $z$ is a periodic point of period $m$ 
or $f^{m-2}(z)=z$. In the last case it follows that $f^{2}(z)=f^{2}\left(f^{m-2}(z)\right)=z$, so $2=m-2$ and a periodic point of period 4 has already been constructed.

$A$ construction of periodic points of periods $6,8,10, \ldots, n-3$. Since $y_{n-1} \notin$ $\left[y_{1}, y_{n-3}\right]$, it follows from Lemma 2.4 that there is a point $t \in\left[y_{n}, y_{n-2}\right)$ such that $f(t)=y_{n-3}$. Let $m=2 s$ be an even integer so that $6 \leq m \leq n-3$. The diagram

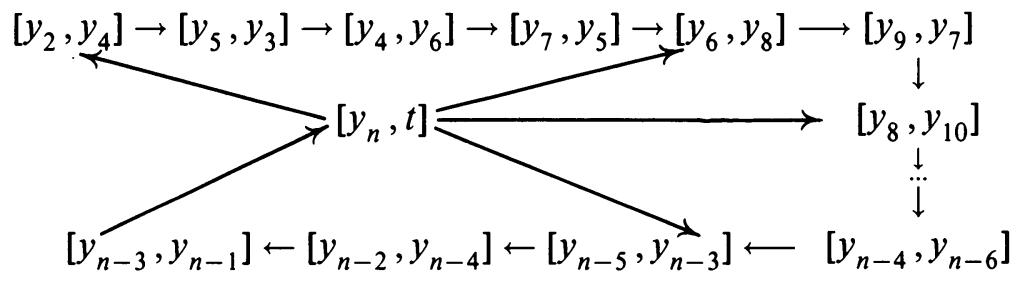

implies that there is a point $z \in\left[y_{n}, t\right]$ such that $f^{2 s}(z)=z, f^{2 s-1}(z) \in$ $\left[y_{n-3}, y_{n-1}\right], f^{2 s-2}(z) \in\left[y_{n-2}, y_{n-4}\right], f^{2 s-3}(z) \in\left[y_{n-5}, y_{n-3}\right], \ldots, f^{2}(z) \in$ $\left[y_{n-2 s+2}, y_{n-2 s}\right]$ and $f(z) \in\left[y_{n-2 s-1}, y_{n-2 s+1}\right]$. By Proposition 5.2 we have that $f^{2 s-1}(z) \neq z$ and $f^{k}(z) \neq z$ for $1 \leq k \leq 2 s-3$. Then either $z$ is a periodic point of period $2 s$ or $f^{2 s-2}(z)=z$. In the last case it follows that $f^{2}(z)=f^{2}\left(f^{2 s-2}(z)\right)=z$, so $2=2 s-2$; then $m=4$ which is smaller than 6 .

Case 3: $n=4$. (Note that in this case we are assuming only that there are no periodic points of odd periods. Periodic points of other even periods may exist.)

Let $I=\left[x_{i}, x_{j}\right]$ be a continuum central with respect to orb $(x)$. By Lemma 4.6 and the assumption that no periodic point of an odd period exists, we get that $f$ sends points of the orbit to the opposite side of $\left(x_{i}, x_{j}\right)$. Thus two points are on each side of $\left(x_{i}, x_{j}\right)$ and we can assume that $I=\left[x_{2}, x_{3}\right]$. Since $f$ does not switch $x_{2}$ and $x_{3}$, at least one of these points is mapped to either $x_{1}$ or $x_{4}$. Without loss of generality we can assume that it is $x_{2}$. Since $x_{2}$ cannot be mapped onto $x_{1}$, we have that $f\left(x_{2}\right)=x_{4}$. Since $f$ sends points of the orbit to the opposite side of $\left(x_{2}, x_{3}\right)$, we have that $f\left(x_{4}\right)=x_{1}, f\left(x_{1}\right)=x_{3}$, and $f\left(x_{3}\right)=x_{2}$.

Suppose that $x_{2} \in\left[x_{3}, x_{4}\right]$. In this case $x_{2}$ would be contained in the same layer of $\left[x_{3}, x_{4}\right]$ as $x_{3}$ and Lemma 4.4 applied with $z=x_{2}$ would contradict the choice of $I$ as a central continuum. Therefore $x_{2} \notin\left[x_{3}, x_{4}\right]$, which implies that $\left[x_{3}, x_{4}\right]$ does not intersect $\left[x_{1}, x_{2}\right)$.

Suppose that $x_{2}$ belongs to the layer of $x_{4}$ in $\left[x_{1}, x_{4}\right]$. In this case $\left[x_{2}, x_{3}\right]$ is contained in the layer of $x_{4}$ in $\left[x_{1}, x_{4}\right]$ and Lemma 4.4 applied with $z=x_{4}$ contradicts the choice of $I$ as a central continuum. So $x_{2}$ is not in the layer of $x_{4}$ in $\left[x_{1}, x_{4}\right]$ and consequently $\left[x_{1}, x_{2}\right]$ does not intersect this layer. Since the layer of $x_{4}$ in $\left[x_{3}, x_{4}\right]$ is contained in the layer of $x_{4}$ in $\left[x_{1}, x_{4}\right]$, we have that $\left[x_{1}, x_{2}\right]$ does not intersect the layer of $x_{4}$ in $\left[x_{3}, x_{4}\right]$.

Now set $a=x_{1}, b=x_{2}, c=x_{3}$ and $d=x_{4}$, and apply Lemma 2.6 to get a periodic point of period 2 . 
Case 4: $n=2^{k} q$, where $k \geq 1$ and $q$ is an odd number (possibly 1 ).

Note that in this case we can assume that $f$ does not have periodic points of odd periods.

Suppose that the theorem is false. Then we can assume that $k$ is the least positive integer so that there is a positive integer $m \triangleleft n=2^{k} q$ and a continuous map from $X$ to $Y$ with a periodic point of period $n$ but no periodic point of period $m$. Since $m \triangleleft n$ and $n$ is even, $m=2 s$ for some integer $s$. Observe that it is enough to consider $m>2$, because the existence of a periodic point of period 4 implies the existence of periodic point of period 2. (See Case 3.) Therefore we can assume that $s \geq 2$.

Consider the function $f^{2}$. $x$ is a periodic point of period $2^{k-1} q$ for $f^{2}$. Note that $s \triangleleft 2^{k-1} q$. By the choice of $k$, there is a point $y$ which is a periodic point of period $s$ for $f^{2}$. Since the orbit of $y$ with respect to $f$ contains at least $s$ distinct points (namely $y, f^{2}(y), \ldots, f^{2 s-2}(y)$ ) and $f^{2 s}(y)=y$, it follows that $y$ is a periodic point of period $2 s$ or $s$ for $f$. If the first is the case the proof is completed. So we can assume that $y$ is a periodic point of period $s$ for $f$. Observe that $s$ is even, because we assumed that $f$ does not have periodic points of odd periods. Since $f^{s}(y)=y$, the period of $y$ under $f^{2}$ is at most $s / 2$. This contradiction completes the proof of the theorem.

\section{REFERENCES}

1. L. Block, J. Guckenheimer, M. Misiurewicz and L. S. Young, Periodic points and topological entropy of one-dimensional maps, Global Theory of Dynamical Systems (Proc. Internat. Conf., Northwestern Univ., 1979), Lecture Notes in Math., vol. 819, Springer, Berlin, 1980, pp. 1834.

2. R. L. Devaney, An introduction to chaotic dynamical systems, Benjamin/Cummings, Menlo Park, Calif., 1980.

3. O. H. Hamilton, A fixed point theorem for pseudo-arcs and certain other continua, Proc. Amer. Math. Soc. 2 (1951).

4. W. T. Ingram, Concerning periodic points in mappings of continua, Proc. Amer. Math. Soc. 104 (1988), 643-649.

5. K. Kuratowski, Topology, Vol. II, Academic Press, New York, Polish Scientific Publishers, Warsaw, 1968.

6. W. Lewis, Periodic homeomorphisms of chainable continua, Fund. Math. 117 (1983), 81-84.

7. L. Mohler, $A$ depth of tranches in $\lambda$-dendroids, Proc. Amer. Math. Soc. 96 (1986), 715-720.

8. A. N. Sarkovskii, Co-existence of cycles of a continuous mapping of the line into itself, Ukrain. Mat. Z. 16 (1964), 61-71. (Russian, English summary)

9. E. S. Thomas, Jr., Monotone decompositions of irreducible continua, Rozprawy Mat. 50 (1960).

Fat Mathematics, Auburn University, Auburn, Alabama 36849 